\title{
Búsqueda de plantas con propiedades insecticidas para el control de Sitophilus zeamais en maíz almacenado
}

\author{
Gonzalo Silva $^{(1)}$, Odette Orrego ${ }^{(1)}$, Ruperto Hepp ${ }^{(1)}$ y Maritza Tapia ${ }^{(1)}$ \\ (1)Universidad de Concepción, Facultad de Agronomía, Departamento de Producción Vegetal, Vicente Méndez 595, Casilla 537, Chillán, Chile. \\ E-mail: gosilva@udec.cl, odetteorrego@latinmail.com, rhepp@udec.cl, martapia@udec.cl
}

\begin{abstract}
Resumen - Se evaluaron, bajo condiciones de laboratorio, 23 plantas en polvo para el control de Sitophilus zeamais Mots. en maíz almacenado. En una primera etapa se evaluaron todos los polvos a una concentración del $1,0 \%(\mathrm{p} / \mathrm{p})$. Posteriormente aquellos polvos con mejores resultados fueron probados en concentraciones del 0,1 , $0,5,1,0$ y $2,0 \%$ en granos de maíz infestados con los insectos a las 24 horas, 30, 60 y 90 días. Se evaluaron 63 tratamientos distribuidos en un diseño experimental completamente al azar y el ensayo se repitió tres veces. En la primera etapa, la mayor mortalidad de insectos se obtuvo con Chenopodium ambrosioides L. y Peumus boldus Mol. con $65,8 \%$ y 99,3\%, respectivamente. Estos tratamientos también propiciaron la menor emergencia de adultos, mientras que la pérdida de peso de los granos no superó el 13,0\%. En las evaluaciones a diferentes concentraciones mostraron una mayor mortalidad y menor emergencia a concentraciones del $1,0 \%$ y $2,0 \%(\mathrm{p} / \mathrm{p})$, obteniéndose para C. ambrosioides una mortalidad del $90,3 \%$ y $90,1 \%$ y para $P$. boldus $97,1 \%$ y $98,8 \%$, respectivamente. La residualidad se mantuvo sólo en el tratamiento de 24 horas.
\end{abstract}

Términos para índice: insecticidas vegetales, cereales, gorgojo del maíz.

\section{Search for plants with insecticidal properties for Sitophilus zeamais control in stored corn}

\begin{abstract}
Twenty three powdered plants were evaluated under laboratory conditions against Sitophilus zeamais Mots. on stored corn. In the first part of the work all plants were evaluated at a concentration of $1.0 \%(\mathrm{w} / \mathrm{w})$. Furthermore the powders with best results were evaluated at concentrations of $0.1,0.5,1.0$ and $2.0 \%$ on corn grains infested with insects during 24 hours, 30, 60 and 90 days. Sixty three treatments in a completely randomized experimental design were evaluated and the study was replicated three times. In the first stage the highest level of insect mortality was exhibited with Chenopodium ambrosioides L. and Peumus boldus Mol. at 1.0\% concentration (w/w), with values of $65.8 \%$ and $99.3 \%$, respectively. The highest insect emergence reductions were obtained with the same treatments and the grain weight was reduced up to $13.0 \%$. In the evaluation with more concentrations, the best results were obtained with treatments at $1.0 \%$ and $2.0 \%(\mathrm{w} / \mathrm{w})$, with C. ambrosioides the insect mortality values were $90.3 \%$ and $90.1 \%$ and with P. boldus mortalities of $97.1 \%$ and $98.8 \%$ were achieved. The residual effect lasted up to 24 hours.
\end{abstract}

Index terms: botanical insecticides, grains, maize weevil.

\section{Introducción}

La conservación y protección de los granos almacenados constituye una necesidad alimenticia, social y económica. Esta conservación se ve amenazada por los insectos que atacan los granos y sus productos durante el almacenamiento (D’Antonio, 1997). Esto es importante en países en desarrollo, donde los productores a pequeña escala ven mermadas sus cosechas a causa de la destrucción de los granos almacenados por roedores, insectos, hongos y bacterias (Larraín, 1994).
La presencia de insectos plaga en granos almacenados trae como consecuencia la pérdida de la calidad del grano tanto para consumo humano como para semilla. En el control de estos, ha sido necesario utilizar en forma intensiva, plaguicidas sintéticos lo cual ha derivado inevitablemente en el surgimiento de resistencia, acumulación en el ambiente e intoxicaciones (Silva et al., 2002). Además por el costo que ello implica, la gran mayoría de los agricultores dedicados a estos cultivos, no utilizan productos químicos por falta de recursos económicos y por los bajos rendimientos que obtienen 
en la agricultura de subsistencia; por lo que se torna obligada la búsqueda de métodos de control de plagas, acorde con la realidad en que viven estos agricultores (Lagunes, 1994).

Existen una serie de métodos de control alternativos que se caracterizan por ser de bajo costo, alta efectividad y factibles de realizar por pequeños agricultores (Braccini \& Picanço, 1995). La revalorización de las plantas como fuente de sustancias con propiedades insecticidas se viene difundiendo desde los últimos 35 años y en algunos países de América Latina como Brasil, México, Ecuador y Chile, se han desarrollado líneas de investigación que buscan en las plantas, compuestos químicos con menor impacto ambiental y potencial para el control de plagas agrícolas (Rodríguez, 2000). El uso de polvos vegetales es una alternativa recuperada de la agricultura de subsistencia y que en evaluaciones con rigor científico han demostrado actuar como repelentes, deterrentes de la oviposición y la alimentación, reguladores de crecimiento e insecticidas tanto de adultos como larvas (Lagunes, 1994).

Existen algunos antecedentes sobre la búsqueda de polvos con propiedades insecticidas contra Acanthoscelides obtectus (Say) (Coleoptera: Bruchidae) (Faroni et al., 1995; Mazzonetto \& Vendramim, 2003; Procopio et al., 2003a), Zabrotes subfasciatus (Boh.) (Coleoptera: Bruchidae) (Oliveira et al., 1999; Procopio et al., 2003a) y Sitophilus zeamais Mots. (Procopio et al., 2003b; Silva et al., 2003), lo que señala las auspiciosas perspectivas del uso de polvos vegetales para la protección de granos en almacenamiento.

El objetivo de la presente investigación fue la búsqueda de plantas con propiedades insecticidas aplicadas en polvo, para el control de Sitophilus zeamais Mots. en maíz almacenado.

\section{Material y Métodos}

Las plantas se colectaron principalmente en la Estación Experimental El Nogal de la Universidad de Concepción, Chillán, Provincia de Nuble, Octava Región, Chile. Se privilegió la colecta de plantas que fueran de ocurrencia frecuente en el medio del agricultor. Estas plantas se secaron en una estufa a $40^{\circ} \mathrm{C}$ durante 48 horas y posteriormente fueron pulverizadas con un molino eléctrico. En el Cuadro 1 se detallan las plantas colectadas y las estructuras utilizadas en las evaluaciones.

Los ejemplares de Sitophilus zeamais M. se obtuvieron de la colonia permanente del Laboratorio de
Toxicología de Insecticidas de la Facultad de Agronomía de la Universidad de Concepción y fueron mantenidas, para su reproducción, en una cámara de cría a una temperatura de $25 \pm 2^{\circ} \mathrm{C}$ y con una fotofase de 16 horas.

Se utilizó la metodología propuesta por Lagunes \& Rodríguez (1989). En la primera etapa todos los polvos vegetales se evaluaron a una concentración del 1\% (p/p). Se eligió esta concentración ya que Lagunes (1994), señala que constituye una concentración razonable al momento de recolectar y utilizar el material vegetal en caso de que la planta resulte efectiva. En frascos de $250 \mathrm{~mL}$ de capacidad se colocaron 100 g de maíz (Zea mays L.). Posteriormente se agregó $1 \mathrm{~g}$ del polvo vegetal a evaluar y se mezcló manualmente de tal forma que el grano quedara cubierto uniformemente. Luego cada frasco se infestó con 10 parejas de $S$. zeamais de no más de siete días de edad para así evitar alteraciones en los resultados por la presencia de la segunda generación de los insectos. La diferenciación de sexos se realizó mediante el criterio de Halstead (1963), quien señala que el rostrum de la hembra es evidentemente más largo y menos ornamentado que el del macho.

Una vez terminada la inoculación todos los frascos fueron puestos en la cámara de cría con las condiciones óptimas para su desarrollo $\left(25^{\circ} \mathrm{C}\right.$ de temperatura y $60 \%$ de humedad relativa). En esta primera parte se evaluaron 24 tratamientos, incluyendo un testigo absoluto formado solamente por el grano y los insectos, con tres repeticiones cada uno.

A los quince días a partir de la infestación con los insectos, éstos fueron extraídos mediante un tamiz y se contabilizó el porcentaje de mortalidad. Este valor fue corregido mediante la fórmula de Abbott (1925) con el objetivo de eliminar la mortalidad natural producida en el testigo y no sobredimensionar el efecto real del tratamiento.

A los 55 días de realizada la infestación se evaluó el porcentaje de emergencia de insectos adultos y el porcentaje de pérdida de peso del grano con relación al testigo absoluto. En el caso de la emergencia de insectos se consideró como $100 \%$ el número de insectos emergidos en el testigo. Para estimar la pérdida de peso de los granos se utilizó la fórmula de Adams \& Schulten (1976): $\mathrm{Pp}=(\mathrm{Ngd} / \mathrm{Ntg}) \times 100 \times \mathrm{C}$,

donde Pp: pérdida de peso [\%], Ngd: número de granos dañados, Ntg: número total de granos, C: 0,125 si el maíz es almacenado como grano suelto o mazorca sin brácteas y 0,222 si el maíz es almacenado como mazorca con brácteas. 
De acuerdo al criterio propuesto por Lagunes (1994), se consideró como prometedor el polvo vegetal que mostrara una mortalidad igual o superior al $50 \%$ y una disminución de la emergencia de un $50 \%$ con relación al testigo absoluto. Los polvos que superaron estos umbrales fueron nuevamente evaluados para los mismos parámetros pero utilizando un mayor número de concentraciones: $0,1 \%, 0,5 \%, 1,0 \%$ y $2,0 \%(\mathrm{p} / \mathrm{p})$.

El efecto residual de los polvos vegetales prometedores se evaluó siguiendo la metodología propuesta por Paez (1987). Se repitió la aplicación de los polvos, en sus respectivas concentraciones, en 1,2 kg de grano que luego se dividieron en 12 submuestras de 100 g cada una. Éstas a su vez se dividieron en cuatro grupos de tres submuestras que fueron infestadas con 10 parejas de insectos a las 24 horas, 30, 60 y 90 días. Posteriormente se evaluó el porcentaje de mortalidad, la emergencia de insectos adultos y la pérdida de peso del grano en los mismos períodos de la primera parte del experimento.

El diseño experimental fue completamente al azar. Para su normalización los valores porcentuales se transformaron previamente a arc sen $\sqrt{x / 100}$. Los datos obtenidos fueron sometidos a un análisis de varianza con el software Stadistical Analysis System (SAS) versión 6.12 (SAS Institute, 1998), y posteriormente, se hizo una comparación de medias mediante el test de Tukey a un nivel de significancia del $5 \%$.

\section{Resultados y Discusión}

De acuerdo al criterio propuesto por Lagunes (1994), sólo 2 de los 23 tratamientos evaluados se pueden considerar como prometedores. Los mejores resultados se obtuvieron con polvos de Chenopodium ambrosioides L. y Peumus boldus Mol. con un 65,8\% y $99,3 \%$ de mortalidad, respectivamente, los cuales mostraron diferencias significativas entre sí (Cuadro 2). Los resultados de $C$. ambrosioides, son similares a los obtenidos por Procopio et al., (2003b) quienes a una concentración de $3 \%(\mathrm{p} / \mathrm{p})$ tuvieron un $100 \%$ de mortalidad de $S$. zeamais. Resultados similares también lograron Silva et al. (2003), quienes obtuvieron una mortalidad de $100 \%$ a una concentración del $1 \%$ (p/p). Por último, Aguilar (1991) señala que esta planta es muy efectiva para el control de $S$. zeamais, información que concuerda ampliamente con los resultados obtenidos.

Experiencias similares para el control de otras plagas de granos almacenados con polvos de $C$. ambrosioides

Cuadro 1. Especies vegetales para determinar su efecto insectistático/insecticida en Sitophilus zeamais Mots.

\begin{tabular}{|c|c|c|c|}
\hline Especie & Familia & Nombre común & Parte utilizada \\
\hline Acacia dealbata Link & Mimosaceae & Aromo & Flor \\
\hline Cersis silicuastrum $\mathrm{L}$. & Cesalpinaceae & Arbol de judea & Hoja y semilla \\
\hline Chenopodium ambrosioides $\mathrm{L}$. & Chenopodiaceae & Paico & Hoja \\
\hline Cotonaster sternianus (Turrill) & Rosaceae & Cotonaster & Fruto \\
\hline Cyperus eragrostis Lam. & Cyperaceae & Chufa & Hoja \\
\hline Datura stramonium $\mathrm{L}$. & Solanaceae & Chamico & Hoja y semilla $^{(1)}$ \\
\hline Daucus carota $\mathrm{L}$. & Apiaceae & Zanahoria silvestre & Flor \\
\hline Erodium moschatum $\mathrm{L}$. & Geraniaceae & Alfilerillo & Hoja \\
\hline Eschscholzia californica Cham. & Papaveraceae & Dedal de oro & Hoja y flor \\
\hline Hypericum perforatum $\mathrm{L}$. & Hypericaceae & Hierba de San Juan & Hoja y flor \\
\hline Lupino angustifolius L. & Fabaceae & Lupino amargo & Fruto \\
\hline Mentha piperita $\mathrm{L}$. & Lamiaceae & Menta & Hoja \\
\hline Peumus boldus Mol. & Monimiaceae & Boldo & Fruto \\
\hline Poa аппиа $\mathrm{L}$. & Hypericaceae & Hierba de San Juan & Fruto \\
\hline Quillaja saponaria Mol. & Rosaceae & Quillay & Hoja \\
\hline Raphanus sativum $\mathrm{L}$. & Monimiaceae & Boldo & Hoja \\
\hline Ruta graveolens L. & Rutaceae & Ruda & Hoja \\
\hline Schinus molle L. & Anacardeaceae & Pimiento boliviano & Fruto \\
\hline Senecio vulgaris L. & Asteraceae & Senecio & Hoja y flor \\
\hline Urtica ureas $\mathrm{L}$ & Urticaceae & Ortiga & Hoja \\
\hline Verbena litorales Kunth & Verbenaceae & Verbena & Hoja y flor \\
\hline Veronica persica Poiret & Scrophulariaceae & Veronica & Hoja y flor \\
\hline
\end{tabular}

${ }^{(1)}$ Evaluadas como tratamientos separados. 
son las de Delobel \& Malonga (1987) los cuales a una concentración de $2,5 \%$ (p/p) utilizaron esta misma planta para el control de Caryedon serratus (Coleoptera: Bruchidae), alcanzando una mortalidad de $90 \%$. A su vez, Mazzonetto \& Vendramim (2003), Oliveira et al. (2003) y Procopio et al. (2003a) consiguieron el $100 \%$ de mortalidad de A. obtectus y Z. subfasciatus.

En cuanto a $P$. boldus, los resultados son similares a los obtenidos por Paez (1987) y Silva et al. (2003), quienes obtuvieron una mortalidad del $100 \%$ y $99,1 \%$, respectivamente, a una concentración del $1 \%(\mathrm{p} / \mathrm{p})$. Estos valores también se asemejan a los de Pizarro (2002) los cuales si bien están dentro del umbral establecido, apenas lo sobrepasaron con un porcentaje de mortalidad de 50,1\%. Además Aguilar (1991) y Lagunes (1994), aunque no señalan valores indican que esta planta es efectiva como polvo vegetal para el control de Sitophilus zeamais.

Los tratamientos que mostraron mayor mortalidad también presentaron una menor emergencia de insectos (Cuadro 2). Con el polvo de C. ambrosioides la emergencia fue de un $11,6 \%$ mientras que con el de
P. boldus no hubo emergencia de insectos. Los resultados de C. ambrosioides están de acuerdo con Delobel \& Malonga (1987), Rodríguez \& Sánchez (1994) y Silva et al. (2003), quienes obtuvieron valores del 0,5\%, $37 \%$ y $0 \%$, respectivamente, los que a pesar de diferir numéricamente entre sí y con el resultado obtenido en este ensayo, están dentro del umbral establecido. Estos resultados también son semejantes a los de Tavares (2002), quien señala que en su investigación la alta mortalidad obtenida con polvos de esta planta fue la causante de las bajas emergencias mostradas por S. zeamais. Por otro lado los valores de P. boldus difieren ligeramente con Pizarro (2002), quien obtuvo una emergencia del $28,8 \%$, pero concuerdan ampliamente con Paez (1987) y Silva et al. (2003) quienes obtuvieron para este mismo parámetro emergencias del orden del $0 \%$ y $0,2 \%$, respectivamente. Además de los tratamientos mencionados, otros 13 mostraron porcentajes de emergencia menores al $50 \%$, por lo que se puede decir que estas plantas presentan un efecto insectistático más que insecticida lo cual es similar a lo señalado por Pizarro (2002) y Silva et al. (2003).

Cuadro 2. Mortalidad y emergencia de insectos adultos y pérdida de peso del grano para el control de Sitophilus zeamais Mots. en granos de maíz almacenados tratados con polvos de especies vegetales al $1 \%{ }^{(1)}$.

\begin{tabular}{|c|c|c|c|}
\hline Especie & $\begin{array}{c}\text { Mortalidad } \\
(\%)\end{array}$ & $\begin{array}{c}\text { Emergencia } \\
(\%)\end{array}$ & $\begin{array}{c}\text { Pérdida de peso } \\
(\%)\end{array}$ \\
\hline Acacia dealbata Link & $0,0 \mathrm{e}$ & $50,0 \mathrm{e}$ & $5,7 b$ \\
\hline Cersis silicuastrum $\mathrm{L}$. & $11,3 \mathrm{c}$ & $35,2 \mathrm{f}$ & $7,1 \mathrm{ab}$ \\
\hline Chenopodium ambrosioides L. & $65,8 b$ & $11,6 \mathrm{ijk}$ & $6,3 \mathrm{ab}$ \\
\hline Cotonaster sternianus (Turrill) & $2,5 \mathrm{de}$ & $26,9 \mathrm{fgh}$ & $5,4 \mathrm{~b}$ \\
\hline Cyperus eragrostis Lam. & $0,0 \mathrm{e}$ & $16,1 \mathrm{hij}$ & $5,5 b$ \\
\hline Datura stramonium L. (hoja) & $1,8 \mathrm{de}$ & $77,7 \mathrm{bc}$ & $6,9 \mathrm{ab}$ \\
\hline Datura stramonium L. (semilla) & $1,8 \mathrm{de}$ & $83,4 b$ & $7,6 \mathrm{ab}$ \\
\hline Daucus carota $\mathrm{L}$. & $3,5 \mathrm{de}$ & $58,1 \mathrm{de}$ & $6,5 \mathrm{ab}$ \\
\hline Erodium moschatum L. & $6,9 \mathrm{~cd}$ & 20,7ghij & $6,0 \mathrm{ab}$ \\
\hline Eschscholzia californica Cham. & $2,9 \mathrm{de}$ & $8,0 \mathrm{jk}$ & $5,7 \mathrm{~b}$ \\
\hline Hypericum perforatum $\mathrm{L}$. & $3,5 \mathrm{de}$ & 16,9 hij & $5,3 \mathrm{~b}$ \\
\hline Lupino angustifolius L. & $0,0 \mathrm{e}$ & $62,9 \mathrm{de}$ & $3,1 b$ \\
\hline Mentha piperita $\mathrm{L}$. & $0,0 \mathrm{e}$ & 22,2 fghij & $12,7 \mathrm{a}$ \\
\hline Peumus boldus Mol. & $99,3 \mathrm{a}$ & $0,0 \mathrm{k}$ & $6,1 \mathrm{ab}$ \\
\hline Poa annua $\mathrm{L}$. & $3,0 \mathrm{de}$ & $79,1 b c$ & $6,3 \mathrm{ab}$ \\
\hline Quillaja saponaria Mol. & $2,9 \mathrm{de}$ & $12,5 \mathrm{hij}$ & $6,3 \mathrm{ab}$ \\
\hline Raphanus sativum L. & $3,3 \mathrm{de}$ & 24,4fghi & $6,6 \mathrm{ab}$ \\
\hline Ruta graveolens L. & $3,5 \mathrm{de}$ & $67,8 \mathrm{~cd}$ & $7,4 \mathrm{ab}$ \\
\hline Schinus molle L. & $1,8 \mathrm{de}$ & 17,4 hij & $5,9 \mathrm{ab}$ \\
\hline Senecio vulgaris $\mathrm{L}$. & $2,3 \mathrm{de}$ & $83,4 b$ & $6,5 \mathrm{ab}$ \\
\hline Urtica ureas $\mathrm{L}$. & $0,6 \mathrm{e}$ & 25,4 fghi & $5,2 b$ \\
\hline Verbena litorales Kunth & $7,2 \mathrm{~cd}$ & $31,6 \mathrm{fg}$ & $4,7 b$ \\
\hline Veronica persica Poiret & $5,9 \mathrm{cde}$ & $60,2 \mathrm{e}^{\circ}$ & $6,2 \mathrm{ab}$ \\
\hline Testigo & - & $100,0 \mathrm{a}$ & $9,5 \mathrm{ab}$ \\
\hline Coeficiente de variación & 20,7 & 10,9 & 33,6 \\
\hline
\end{tabular}

${ }^{(1)}$ Tratamientos con igual letra en la columna, no difieren estadísticamente por el test de Tukey $(\alpha=0,05)$. 
Se esperaría que con una mayor mortalidad y una baja emergencia exista también una menor pérdida de peso del grano. En esta investigación esto no ocurrió completamente ya que si bien es cierto en los granos tratados con $C$. ambrosioides, ésta indujo una pérdida de peso de 6,3\%, mientras que en Silva et al. (2003) este valor fue de $0,04 \%$, lo cual difiere con los resultados obtenidos, se mantiene una tendencia de menores valores de pérdida de peso en comparación al testigo. En cuanto al efecto causado por P. boldus, en general se produjo una baja pérdida de peso no existiendo diferencias estadísticamente significativas con la mayoría de los tratamientos restantes (Cuadro 2). Esto difiere con Pizarro (2002) quien al 1\% (p/p) obtuvo una pérdida de peso de $1,7 \%$, lo cual es menor a lo obtenido en la presente investigación que fue de $6,1 \%$. A su vez Silva et al. (2003) obtuvo una pérdida de peso del grano de $0,09 \%$ que también es menor al valor experimental obtenido. Estos resultados podrían explicarse en base a que algunas plantas son repelentes y para este caso en particular podría deberse a un efecto disuasivo de la alimentación u ovoposición (Coats, 1994).

C. ambrosioides y $P$. boldus se evaluaron a concentraciones de $0,1 \%, 0,5 \%, 1,0 \%$ y $2 \%(\mathrm{p} / \mathrm{p})$, pero sólo las concentraciones de $1,0 \%$ y $2,0 \%$ (p/p) produjeron una alta mortalidad (Cuadro 3). Así C. ambrosioides, propició una mortalidad de $90,3 \%$ y $90,1 \%$ al $1 \%$ y $2 \%$ $(\mathrm{p} / \mathrm{p})$, respectivamente, valores que no son diferentes estadísticamente entre sí. En cuanto a $P$. boldus el polvo de esta planta al $1,0 \%$ y $2,0 \%$ (p/p) mostró una mortalidad del $97 \%$ y $98,8 \%$, respectivamente, no siendo tampoco diferentes estadísticamente entre sí. Los resultados obtenidos a la concentración de 2,0\% (p/p) concuerdan con Pizarro (2002) quien reporta una mortalidad de $82,8 \%$. Estos resultados son considerablemente mayores al registrado en la primera etapa de este ensayo que fue de $65,8 \%$, lo cual reafirma la variabilidad que presentan los insecticidas vegetales ya que el efecto de un plaguicida vegetal, debido al contenido de sus compuestos activos, depende de factores como la especie y variedad de la planta, época de recolección, órgano cosechado, forma de preparación y de extracción, aplicación y la influencia del ambiente (Silva et al., 2002).

La emergencia de $S$. zeamais en los tratamientos evaluados con C. ambrosioides fue de $4,0 \%$ y $3,8 \%$ al $1 \%$ y $2 \%$ (p/p) (Cuadro 3), resultados que difieren con los obtenidos en la primera etapa de esta investigación que fue de $11,6 \%$ al $1 \%(\mathrm{p} / \mathrm{p})$, valor que también difiere con Silva et al. (2003) quien a la misma concentración no obtuvo emergencia de insectos. En cuanto a $P$. boldus la emergencia disminuyó considerablemente, con resultados al $1,0 \%$ y $2,0 \%(\mathrm{p} / \mathrm{p})$ de $0,1 \%$ y $0 \%$ de emergencia de insectos adultos, respectivamente. Estos resultados son semejantes a los obtenidos en la primera etapa de este ensayo en donde al $1 \%(\mathrm{p} / \mathrm{p})$ no se obtuvo emergencia de insectos, y también concuerda con Paez (1987) y Silva et al. (2003) con valores de $0 \%$ y $0,2 \%$, respectivamente, a la misma concentración. Estos resultados difieren levemente con lo obtenido por Pizarro (2002) a la concentración de $2 \%$ (p/p), quien obtuvo una emergencia de $5,5 \%$.

El grano tratado con C. ambrosioides al $1 \%$ y $2 \%$ $(\mathrm{p} / \mathrm{p})$ presentó los menores porcentajes de pérdida de peso del grano con un $5,3 \%$ y $2,7 \%$, respectivamente, mientras que las concentraciones de $0,1 \%$ y $0,5 \%(\mathrm{p} / \mathrm{p})$

Cuadro 3. Mortalidad y emergencia de insectos adultos y pérdida de peso del grano de maíz para el control de Sitophilus zeamays Mots. con polvos de Chenopodium ambrosioides L. y Peumus boldus Mol. a diferentes concentraciones ${ }^{(1)}$.

\begin{tabular}{|c|c|c|c|c|}
\hline Concentración (\%) & Especie & $\begin{array}{c}\text { Mortalidad } \\
(\%)\end{array}$ & $\begin{array}{c}\text { Emergencia } \\
(\%)\end{array}$ & $\begin{array}{c}\text { Pérdida de peso } \\
(\%)\end{array}$ \\
\hline 0,1 & C. ambrosioides & $3,1 \mathrm{c}$ & $85,6 b$ & $8,8 \mathrm{a}$ \\
\hline 0,5 & & $12,9 b$ & $78,0 \mathrm{~b}$ & $8,0 \mathrm{~b}$ \\
\hline 1,0 & & $90,3 \mathrm{a}$ & $4,0 \mathrm{c}$ & $5,3 \mathrm{c}$ \\
\hline 2,0 & & $90,1 \mathrm{a}$ & $3,8 \mathrm{c}$ & $2,7 \mathrm{~d}$ \\
\hline Testigo & & & $100,0 \mathrm{a}$ & $8,2 \mathrm{ab}$ \\
\hline Coeficiente de variación & & 3,0 & 6,9 & 4,2 \\
\hline 0,1 & P. boldus & $5,3 \mathrm{c}$ & $77,9 \mathrm{~b}$ & $8,3 a$ \\
\hline 0,5 & & $41,9 \mathrm{~b}$ & $59,8 \mathrm{c}$ & $7,1 \mathrm{~b}$ \\
\hline 1,0 & & $97,1 \mathrm{a}$ & $0,1 \mathrm{~d}$ & $1,6 \mathrm{c}$ \\
\hline 2,0 & & $98,8 \mathrm{a}$ & $0,0 \mathrm{~d}$ & $0,14 \mathrm{~d}$ \\
\hline Testigo & & & $100,0 \mathrm{a}$ & $8,2 \mathrm{a}$ \\
\hline Coeficiente de variación & & 4,2 & 5,2 & 3,8 \\
\hline
\end{tabular}

${ }^{(1)}$ Tratamientos con igual letra en la columna, no difieren estadísticamente por el test de Tukey $(\alpha=0,05)$. 
superaron el 8,0\%, siendo significativamente diferentes entre sí pero no con el testigo (Cuadro 3). Por su lado, P. boldus tuvo una pérdida de peso de $1,6 \%$ y $0,14 \%$ al $1 \%$ y $2 \%$ (p/p), respectivamente, mostrando diferencias estadísticamente significativas entre sí y con el testigo que alcanzó un 8,2\%. Estos resultados están de acuerdo a lo obtenido por Pizarro (2002) quien para este parámetro al $1 \%$ y $2 \%(\mathrm{p} / \mathrm{p})$ señala pérdidas de peso de $1,7 \%$ y $1,0 \%$. Por otra parte, Silva et al. (2003) usando este polvo a una concentración del $1 \%(\mathrm{p} / \mathrm{p})$ obtuvo pérdidas de peso de $0,09 \%$.

Se pudo observar que las plantas con resultados prometedores sólo mantuvieron su efectividad a las 24 horas de realizada la infestación (Cuadro 4). En el caso de C. ambrosioides los resultados obtenidos siguieron la tendencia señalada ya que al $1,0 \%$ y $2,0 \%(\mathrm{p} / \mathrm{p})$ solo en el tratamiento de 24 horas se obtuvo una mortalidad de $85,2 \%$ y $100 \%$, valores diferentes estadísticamente entre sí. Estos resultados difieren con Aguilar (1991) quien nuevamente no señala valores, pero indica que si bien es cierto esta planta no elimina a todos los insectos, protege el maíz durante 3 a 4 meses. Para P. boldus la mortalidad alcanzó un $95 \%$ y $100 \%$ al $1,0 \%$ y $2,0 \%$ $(\mathrm{p} / \mathrm{p})$, respectivamente, sin presentar diferencias significativas entre sí a las 24 horas. A los 30, 60 y 90 días a estas mismas concentraciones, la mortalidad no superó el 4,0\% en ninguno de los tratamientos. Esto concuerda con Paez (1987) quien al 1,0\% (p/p) a las 24 horas obtuvo una mortalidad del $100 \%$, mientras que para el resto de los tratamientos los valores no superaron el $10 \%$. Sin embargo, este resultado difiere con lo señalado por
Aguilar (1991), quien menciona que con P. boldus se puede proteger el maíz hasta por un año, aunque no señala a qué concentraciones.

En la emergencia de insectos adultos los resultados se mostraron semejantes a los bioensayos anteriores ya que para C. ambrosioides ésta alcanzó $10,8 \%$ y $3,6 \%$ al $1,0 \%$ y $2,0 \%(\mathrm{p} / \mathrm{p})$ a las 24 horas, mientras que en el resto de los tratamientos promedió una emergencia de insectos de $74 \%$ (Cuadro 4). En cuanto a P. boldus a las 24 horas la emergencia fue de $4,7 \%$ y $0 \%$ al $1,0 \%$ y $2,0 \%(\mathrm{p} / \mathrm{p})$, mientras que para el resto de los tratamientos el promedio fue de $71 \%$.

El porcentaje de pérdida de peso en ambos casos sólo se vio afectado en el tratamiento realizado a las 24 horas siendo estadísticamente diferente entre sí, con el testigo y con la mayoría de los tratamientos restantes para ambas especies vegetales (Cuadro 4). Así, el grano tratado con C. ambrosioides, a las mismas concentraciones, fue de $6,9 \%$ y $3,1 \%$ respectivamente siendo diferentes significativamente entre sí, mientras que el resto de los tratamientos osciló entre $5,0 \%$ y $8,5 \%$. En cambio para los granos mezclados con polvo de $P$. boldus al $1,0 \%$ y $2,0 \%(\mathrm{p} / \mathrm{p})$ presentó una pérdida de peso de $2,4 \%$ y $1,1 \%$ mientras el resto de los tratamientos tuvieron en promedio una pérdida de peso de $8,0 \%$. Es muy probable que este efecto reducido en el tiempo se deba a las características que presentan las sustancias de origen natural como son la inestabilidad en el medio ambiente ya que son sustancias altamente biodegradables, cuyos principios activos se metabolizan rápidamente ante la radiación solar, humedad

Cuadro 4. Mortalidad y emergencia de insectos adultos y pérdida de peso del grano para el control de Sitophilus zeamays Mots. en los tratamientos de residualidad con granos de maíz tratados con polvo de Peumus boldus Mol. (PB) y Chenopodium ambrosioides L. (CA) al $1 \%$ y $2 \%(1)$.

\begin{tabular}{|c|c|c|c|c|c|c|c|}
\hline \multirow[t]{2}{*}{ Residualidad } & \multirow[t]{2}{*}{ Tratamiento } & \multicolumn{2}{|c|}{ Mortalidad (\%) } & \multicolumn{2}{|c|}{ Emergencia (\%) } & \multicolumn{2}{|c|}{ Pérdida de peso $(\%)$} \\
\hline & & PB & $\mathrm{CA}$ & PB & $\mathrm{CA}$ & PB & $\mathrm{CA}$ \\
\hline \multirow[t]{3}{*}{24 horas } & Testigo & $0,0 \mathrm{~b}$ & $0,9 \mathrm{~g}$ & $100,0 \mathrm{a}$ & $100,0 \mathrm{a}$ & $8,2 b$ & $10,0 \mathrm{a}$ \\
\hline & $1 \%$ & $95,0 \mathrm{a}$ & $85,2 \mathrm{~b}$ & $4,7 \mathrm{e}$ & $10,8 \mathrm{e}$ & $2,4 d$ & $6,9 \mathrm{e}$ \\
\hline & $2 \%$ & $100,0 \mathrm{a}$ & $100,0 \mathrm{a}$ & $0,0 \mathrm{e}$ & $3,6 \mathrm{e}$ & $1,1 \mathrm{e}$ & $3,1 \mathrm{~g}$ \\
\hline \multirow[t]{3}{*}{30 días } & Testigo & $0,0 \mathrm{~b}$ & $2,8 f g$ & $100,0 \mathrm{a}$ & $100,0 \mathrm{a}$ & $9,4 a$ & $7,0 \mathrm{e}$ \\
\hline & $1 \%$ & $1,7 \mathrm{~b}$ & $20,9 c$ & $65,1 d$ & $96,2 \mathrm{ab}$ & $8,2 b$ & $7,2 \mathrm{de}$ \\
\hline & $2 \%$ & $3,9 b$ & $23,7 \mathrm{c}$ & $72,9 \mathrm{bc}$ & $81,1 \mathrm{c}$ & $8,9 \mathrm{a}$ & $8,0 \mathrm{~cd}$ \\
\hline \multirow[t]{3}{*}{60 días } & Testigo & $2,3 b$ & $1,1 \mathrm{~g}$ & $100,0 \mathrm{a}$ & $100,0 \mathrm{a}$ & $9,3 \mathrm{a}$ & $9,2 \mathrm{ab}$ \\
\hline & $1 \%$ & $1,6 b$ & $10,7 d$ & $75,4 b$ & $89,8 b c$ & $7,5 \mathrm{c}$ & $7,2 \mathrm{de}$ \\
\hline & $2 \%$ & $1,3 b$ & $21,9 \mathrm{c}$ & $66,4 \mathrm{~cd}$ & $64,8 \mathrm{~d}$ & $8,2 \mathrm{~b}$ & $8,5 \mathrm{bc}$ \\
\hline \multirow[t]{3}{*}{90 días } & Testigo & $0,0 \mathrm{~b}$ & $0,0 \mathrm{~g}$ & $100,0 \mathrm{a}$ & $100,0 \mathrm{a}$ & $9,2 \mathrm{a}$ & $5,1 \mathrm{f}$ \\
\hline & $1 \%$ & $1,7 \mathrm{~b}$ & $6,1 \mathrm{ef}$ & $75,5 b$ & $56,3 \mathrm{~d}$ & $7,2 \mathrm{c}$ & $5,8 f$ \\
\hline & $2 \%$ & $3,3 \mathrm{~b}$ & 8,9de & $74,5 \mathrm{~b}$ & $58,0 \mathrm{~d}$ & $8,2 \mathrm{~b}$ & $5,3 \mathrm{f}$ \\
\hline Coeficiente de variación (\%) & & 10,4 & 6,6 & 3,5 & 4,2 & 2,9 & 4,7 \\
\hline
\end{tabular}

${ }^{(1)}$ Tratamientos con igual letra en la columna, no difieren estadísticamente por el test de Tukey $(\alpha=0,05)$. 
microclimática y el ataque microbiano, reduciendo su acción en el tiempo y haciendo necesarias aplicaciones frecuentes (Morales \& García, 2000).

\section{Conclusiones}

1. Chenopodium ambrosioides L. y Peumus boldus Mol. son efectivos controladores de Sitophilus zeamais Mots. en maíz almacenado.

2. El efecto residual para Chenopodium ambrosioides L. y Peumus boldus Mol. en polvo es menor a 30 días.

\section{Referencias}

ABBOTT, W.S. A method for computing the effectiveness of an insecticide. Journal of Economic Entomology, v.18, p.265-267, 1925.

ADAMS, J.M.; SCHULTEN, G.G.M. Losses caused by insects, mites and microorganisms. In: American Association of Cereal Chemists. Postharvest grain loss assessment methods. Slough, England, 1976. p.83-93.

AGUILAR, J. Consejos para almacenar el maíz en casa. México DF: Ediciones Libro del Rincón, 1991. 95p.

BRACCINI, A.; PICANÇO, M. Manejo integrado de pragas do feijoeiro no armazenamento. Revista Brasileira de Armazenamento, v.20, p.37-43, 1995.

COATS, J.R. Risks from natural versus synthetic insecticides. Annual Review of Entomology, v.39, p.489-515, 1994.

D’ANTONIO, L. Principais pragas de grãos armazenados. In: CONGRESO BRASILEIRO DE ENGENHERIA AGRÍCOLA, 26., 1997, Campina Grande, Paraíba. Armazenamento de grãos e sementes nas propiedades rurais. Campina Grande, 1997. p.189-291.

DELOBEL, A.; MALONGA, P. Insecticidal properties of six plant materials against Caryedon serratus (OL.) (Coleoptera:Bruchidae). Journal of Stored Product Research, v.23, p.173-176, 1987.

FARONI, L.; MOLIN, L.; ANDRADE, E.; CARDOSO, E. Utilização de productos naturais no controle de Acanthoscelides obtectus em feijão armazenado. Revista Brasileira de Armazenamento, v.20, p.44-48, 1995.

HALSTEAD, D.G.H. External sex differences in stored-products coleoptera. Bulletin of Entomological Research, v.54, p.119-134, 1963.

LAGUNES, A. Extractos de polvos vegetales y polvos minerales para el combate de plagas del maíz y del frijol en la agricultura de subsistencia. México: Colegio de Postgraduados/USAID/ CONACYT/BORUCONSA, 1994. 35p.

LAGUNES, A.; RODRÍGUEZ, C. Búsqueda de tecnología apropiada para el combate de plagas del maíz almacenado en condiciones rústicas. Montecillo, México: CONACYT/Colegio de Postgraduados, 1989. 150p.

LARRAÍN, P. Manejo integrado de plagas en granos almacenados. Investigación y Progreso Agropecuario, v.81, p.10-16, 1994.

MAZZONETTO, F.; VENDRAMIM, J. Efeito de pós de origen vegetal sobre Acanthoscelides obtectus (Say) (Coleoptera:Bruchidae) em feijão armazenado. Neotropical Entomology, v.32, p.145-149, 2003.

MORALES, S.; GARCÍA, C.M. Metodología para la evaluación del potencial insecticida de especies forestales. Revista Facultad Nacional Agronomía, v.53, p.787-800, 2000.

OLIVEIRA, J. de; VENDRAMIM, J.; HADDAD, M. Bioatividade de pós vegetais sobre o caruncho do feijão armazenados. Revista de Agricultura, v.4, p.44-48,1999.

PAEZ, A. Uso de polvos vegetales e inertes minerales como una alternativa para el combate del gorgojo del maíz Sitophilus zeamais Motschulsky (Coleoptera: Curculionidae) en maíz almacenado. 1987. 135p. Tesis (Magíster en Ciencias) - Colegio de Postgraduados en Ciencias Agrícolas, Montecillo, México.

PIZARRO, D.I. Control de Sitophilus zeamais Motschulsky con plantas medicinales en polvo. 2002. 30p. Tesis (Ingeniero Agrónomo) - Universidad de Concepción, Chillán.

PROCOPIO, S.; VENDRAMIM, J.; RIBEIRO, J.; SANTOS, J. dos. Bioactividade de diversos pós de origen vegetal em relação a Sitophilus zeamais Mots. (Coleoptera: Curculionidae). Ciência Agrotecnica, v.27, p.1231-1236, 2003b.

PROCOPIO, S.; VENDRAMIM, J.; RIBEIRO, J.; SANTOS, J. dos. Efeito de pós vegetais sobre Acanthoscelides obtectus (SAY) e Zabrotes subfasciatus (BOH.) (Colepotera: Curculionidae). Revista Ceres, v.50, p.395-405, 2003a.

RODRÍGUEZ, D.A.; SÁNCHEZ. S. Polvos vegetales para el combate de Sitophilus zeamais y Zabrotes subfasciatus en maíz y frijol. Turrialba, v.44, p.160-167, 1994.

RODRÍGUEZ, H.C. Plantas contra plagas: potencial práctico de ajo, anona, nim, chile y tabaco. Texcoco, México: Red de Acción sobre Plaguicidas y Alternativas en México (RAPAM), 2000. 133p.

SAS INSTITUTE (Cary, Estados Unidos). Language guide for personal computers release, 6.03 ed. Cary, 1998. 1028p.

SILVA, G.; LAGUNES, A.; RODRÍGUEZ, J.; RODRÍGUEZ, D. Evaluación de polvos vegetales solos y en mezcla con carbonato de calcio para el control de Sitophilus zeamais Motschulsky en maíz almacenado. Ciencia e Investigación Agraria, v.30, p.153-160, 2003.

SILVA, G.; LAGUNES, A.; RODRÍGUEZ, J.; RODRÍGUEZ, D. Insecticidas vegetales: una vieja y nueva alternativa para el manejo de plagas. Manejo Integrado de Plagas y Agroecología, v.66, p.412, 2002.

TAVARES, M.A.G.C. Bioatividade da erva-de-santa-maria, Chenopodium ambrosioides $\mathrm{L}$. (Chenopodiaceae), em relação a Sitophilus zeamais (Col.: Curculionidae). 2002. 59p. Tese (Mestrado) - Universidade de São Paulo, Piracicaba.

Recibido el 11 de febrero de 2004 y aceptado el 21 de septiembre de 2004 